\title{
Analysis of general rate model of linear chromatography considering finite rates of the adsorption and desorption steps
}

\author{
Shamsul Qamar ${ }^{\mathrm{a}, \mathrm{b}, *}$, Noreen Akram ${ }^{\mathrm{a}}$, Andreas Seidel-Morgenstern ${ }^{\mathrm{b}}$ \\ ${ }^{a}$ Department of Mathematics, COMSATS Institute of Information Technology, Islamabad, Pakistan \\ ${ }^{b}$ Max Planck Institute for Dynamics of Complex Technical Systems Magdeburg, Germany
}

\begin{abstract}
This article presents semi-analytical solutions and temporal moments of the general rate model of chromatography with a focus on evaluating the effect of finite rates for the adsorption and desorption steps, typically considered to be in equilibrium. The model equations are analytically solved in the Laplace domain and numerical Laplace inversion is applied to get back solutions in the actual time domain. The expression of first four temporal moments are derived from the analytical solutions in the Laplace domain. The derived analytical solutions and moments are helpful tools to predict dynamic behaviors inside the column and to evaluate the influence of model parameters on the elution profiles, in particular the effect of finite rates of the intrinsic adsorption and desorption steps. The correctness of analytical solutions are verified through the numerical solutions of a high resolution finite volume scheme. Several case studies are considered to quantify effects of the rate constants for adsorption and desorption, axial dispersion, film mass transfer resistance, intraparticle diffusion resistance, and inlet boundary conditions on the elution profiles.
\end{abstract}

Keywords: Chromatography, general rate model, dynamic sorption kinetics, analytical solutions, moment analysis.

\footnotetext{
*Corresponding author. Tel: +49-391-6110454; fax: +49-391-6110500

Email address: qamar@mpi-magdeburg.mpg.de (Shamsul Qamar)
} 


\section{Introduction}

Chromatography is known as one of the powerful separation and purification techniques used in petrochemical, fine chemical, pharmaceutical, biotechnical and food industries. For instance, this technique is applied to separate chiral molecules, enzymes, sugar and to purify proteins or to produce insulin. It has capability to rapidly produce milligrams to tons of purified products. In particular, this technology is highly effective within those process industries where because of physicochemical limitations traditional separation operations such as distillation, solvent extraction, crystallization, evaporation are not applicable. In the last few decades, this technique has achieved considerable industrial popularity, see e.g. Ruthven (1984); Guiochon and Lin (2003); Guiochon et al. (2006).

Chromatographic techniques are based on selective adsorption of mixture compounds on a solid phase (or liquid phase) with high surface area. In liquid-solid column chromatography, a mobile (liquid) phase, carrying mixture components is passed through a tabular column containing the stationary (solid) phase. During migration, each component of the mixture interact to varying degrees with the stationary phase. Thus, components of the mixture are continuously partitioned between solid (adsorbent) and mobile phases. Components having strong interaction with the stationary phase propagate slowly along the column compared to the weakly interacting components. A complete separation of the components can be achieved if the column length is long enough Ruthven (1984); Guiochon et al. (2006). Other applications of adsorption phenomena can be found in Lemlikchi et al. (2015); Kitous et al. (2016).

Mathematical modeling plays an important role in the design, understanding, and optimization of several chemical engineering processes. Modeling of the chromatographic columns are helpful to understand the transport mechanism and to approximate chemicalphysical parameters from the analysis of experimental data. It offers a technique for predicting the dynamic behaviors of the solute in the columns without carrying out expensive and time-consuming experiments in the laboratories. The numerical tools are often more valuable than the conventional experimental methods in terms of providing more 
profound insights and complete information that cannot be directly measured or observed, see Guiochon et al. (2006); Schmidt-Traub et al. (2012). A common practice in the mathematical modeling of chromatographic system is to consider a column packed with mono dispersed spherical particles and to write unsteady-state mass balances of both the mobile and the stationary phases and to impose some simplifying assumptions incompliance with the physics of the system. This leads to a system of partial differential equations (PDEs) coupled with some algebraic and/or differential equations.

In the literature, a number of dynamic models have been introduced to simulate the chromatographic process, see for example Ruthven (1984); Guiochon and Lin (2003); Guiochon et al. (2006). Each model considers a different level of complexity to describe the process. The most important to mention are the equilibrium dispersive model (EDM), the lumped kinetic model (LKM), and the general rate model (GRM). The general rate model (GRM) is regarded as the most comprehensive model for the simulation of fixed-bed adsorbers. In this model, the axial dispersion and all the mass-transfer resistances are taken into account, such as the external mass transfer of adsorbate from the bulk phase to the external surface of the adsorbent, the diffusion transport through the pores of the adsorbent, and the adsorption-desorption kinetics at the active sites. Herby, the latter aspect is studied less frequently. In most models applied to simulate chromatographic processes, permanently established adsorption equilibria are assumed. The GRM can be used to study the effects of different mass-transfer kinetics on the elution profiles. Either analytical or numerical solution techniques can be used to solve the model equations, depending on the degree of tractability of the governing system of equations.

The analytical solutions of the packed-beds for mono-dispersed solid spherical particles dates back to the pioneering works of Hougen and Marshal (1947), Thomas (1948, 1951), and Rosen et al. (1952). Afterwards, several other researchers presented analytical solutions of different models for fixed-bed absorber, see e.g. Rasmuson (1985); Carta (1988); Li et al. (2003, 2004); Guiochon et al. (2006); Shams (2014) and references therein.

The analytical solutions of different chromatographic models in the Laplace domain have been recently derived considering the assumption of a linear isotherm, see e.g. Felinger and 
Guiochon (2004); Javeed et al. (2013); Miyabe (2014); Qamar et al. (2014, 2015). When the Laplace transform is applied in terms of time variable, a function of time is transformed into a function of complex frequency. Now, the phenomenon is converted to the Laplace domain in which certain operations can be carried out, for example temporal moments can be found using the Laplace transformed function. After deriving the solution in the Laplace domain, it requires a back transformation to the actual time domain. However, the analytical back transformation is not possible in all situations, for example in the current situation of GRM. Thus, the numerical Laplace inversion is applied in such a scenario, see Rice et al. (1995).

The temporal moment analysis is a useful technique to extract information about the retention equilibrium and the mass transfer kinetics in the column. It provides accurate information about band broadening caused by several mass transfer kinetic processes. The technique has been comprehensively discussed in the literature, see instance Kubin (1965a,b); Kucera (1965); Schneider and Smith (1968); Suzuki (1973); Wolff et al. (1979, 1980); Ruthven (1984); Lenhoff (1987); Gengliang and Zhide (1996); Antos (2003); Guiochon et al. (2006), Miyabe et al. (2003, 2007, 2009)

This study extends our previous analysis of general rate model from quasi-stationary sorption model to dynamic linear sorption kinetic model, see Qamar et al. (2014). The previous model is a limiting case of the current model when the adsorption rate constant is very large. Analytical solutions are derived for the two sets of boundary conditions (BCs), i.e. the Dirichlet and Danckwers BCs. For further analysis, the first four temporal moments are derived from the Laplace transformed solutions. The analytical expressions of height equivalent to theoretical plate (HETP) are derived for both sets of BCs. The influences of adsorption and desorption rates, axial dispersion, film mass transfer resistance, intraparticle diffusion resistance, and inlet boundary conditions on the elution profiles are discussed and analyzed.

The current study also extends and generalizes the recent work of Miyabe (2014) who already analyzed GRM with finite adsorption and desorption rates. The major novelty of this article include: (a) the derivation of analytical solutions for both Dirichlet and 
Danckwerts BCs considering pulse injections of finite widths, (b) the derivation of analytical expressions for the first four temporal moments and equations of HETP curve for the considered two sets of boundary conditions, (d) consideration of different case studies to quantify effects of the rate constants for adsorption and desorption, axial dispersion, film mass transfer resistance, intraparticle diffusion resistance, and inlet boundary conditions on the elution profiles, (c) Correction of Eq. (3) of Miyabe (2014) by incorporating the pore and surface diffusions in appropriate manner, see Eq. (2) of this manuscript.

\section{The GRM with finite adsorption and desorption rates}

This study considers an isothermal adsorption column packed with porous particles. Concentration pulses of an adsorbate are periodically injected to a flowing stream passing through the column. The adsorption column is subjected to axial dispersion, external mass-transfer resistance, intraparticle diffusion resistance, and adsorption-desorption kinetics.

Based on the aforementioned assumptions, the mass balance for the bulk phase is given as

$$
\frac{\partial C_{b}}{\partial t}+u \frac{\partial C_{b}}{\partial z}=D_{b} \frac{\partial^{2} C_{b}}{\partial z^{2}}-\frac{3 F k_{\mathrm{ext}}}{R_{p}}\left(C_{b}-\left.C_{p}\right|_{r=R_{p}}\right) .
$$

In the above equation, $C_{b}$ and $C_{p}$ are the concentrations of a solute in the bulk of the fluid and in particle pores, respectively. The phase ratio $F$ is defined as $F=\left(1-\epsilon_{e}\right) / \epsilon_{e}$, where $\epsilon$ is the external porosity. Moreover, $u$ is the interstitial velocity, $D_{b}$ represents the axial dispersion coefficient, $k_{\text {ext }}$ is the external mass transfer coefficient, $R_{p}$ is the radius of the adsorbent, and $t$ and $z$ denote time and axial coordinate of the column. In addition, $r$ denotes the radial coordinate

The mass balance equation for the solute in the stationary phase can be expressed assuming two mechanisms of intraparticle transport, namely pore and surface diffusions:

$$
\epsilon_{p} \frac{\partial C_{p}}{\partial t}+\left(1-\epsilon_{p}\right) \frac{\partial Q_{p}}{\partial t}=\frac{1}{r^{2}} \frac{\partial}{\partial r}\left(r^{2}\left[\epsilon_{p} D_{p} \frac{\partial C_{p}}{\partial r}+\left(1-\epsilon_{p}\right) D_{s} \frac{\partial Q_{p}}{\partial r}\right]\right)
$$

where $Q_{p}$ is the non-equilibrium concentration of the solute in the stationary phase, $\epsilon_{p}$ is the internal porosity, $D_{p}$ is the pore diffusivity, and $D_{s}$ is the surface diffusivity. 
In this study, linear adsorption kinetics of finite rate are assumed. The following mass balance for the amount of the solute adsorbed on the stationary phase is used

$$
\frac{\partial Q_{p}}{\partial t}=K_{d}\left(a C_{p}-Q_{p}\right)
$$

where the well-known Henry's constant $a$, used in linear equilibrium models, is defined as

$$
a=\frac{K_{a}}{K_{d}}
$$

In the above equations, $K_{a}$ and $K_{d}$ denote the adsorption and desorption rate constants, respectively. Eq. (3) is a simplified adsorption-desorption kinetic expression assuming that solute loading is sufficiently low at all times so that the number of available sites for adsorption does not change as the solute moves through the column. For rapid desorption (and adsorption) steps, i.e. $K_{d} \rightarrow \infty$, Eq. (3) gives $Q_{p}=a C_{p}$. In that case, the current model reduces to the linear GRM recently analyzed by Qamar et al. (2014).

In the context of this work, we will study in particular the impact of finite $K_{d}$-values on the course of elution profiles. Eqs. (1) and (2) are connected at $r=R_{p}$ via the following expression that quantifies the temporal change of the average loading of the particles:

$$
\left[\epsilon_{p} D_{p} \frac{\partial C_{p}}{\partial r}+\left(1-\epsilon_{p}\right) D_{s} \frac{\partial Q_{p}}{\partial r}\right]_{r=R_{p}}=k_{\mathrm{ext}}\left(C_{b}-\left.C_{p}\right|_{r=R_{p}}\right) .
$$

To simply the notations and reduce the number of variables, the following dimensionless quantities are introduced:

$$
\begin{aligned}
& c_{b}=\frac{C_{b}}{C_{\mathrm{inj}}}, c_{p}=\frac{C_{p}}{C_{\mathrm{inj}}}, q_{p}=\frac{Q_{p}}{a C_{\mathrm{inj}}} \tau=\frac{u t}{L}, \rho=r / R_{p}, \quad x=\frac{z}{L}, P e=\frac{L u}{D_{b}}, \eta_{p}=\frac{D_{p} L}{u R_{p}^{2}}, \\
& \eta_{s}=\frac{D_{s} L}{u R_{p}^{2}}, B i_{p}=\frac{k_{\mathrm{ext}} R_{p}}{D_{p}}, B i_{s}=\frac{k_{\mathrm{ext}} R_{p}}{D_{s}}, \xi=3 F \frac{k_{\mathrm{ext}}}{R_{p}} \frac{L}{u} .
\end{aligned}
$$

Moreover, a dimensionless desorption rate is expressed as

$$
k_{\mathrm{d}}=\frac{K_{d} L}{u}
$$

In Eq. (6a), $C_{\text {inj }}$ denotes the non-zero injected bulk concentration, $P e$ is the Peclet number based on column length, $B i$ represents modified Biot number, and $\eta_{p}$ and $\eta_{s}$ describe 
the ratio of space time and interaparticle diffusion times. Using the above dimensionless variables, the model Eqs. (1) to (3) can be rewritten as

$$
\begin{gathered}
\frac{\partial c_{b}}{\partial \tau}+\frac{\partial c_{b}}{\partial x}=\frac{1}{P e} \frac{\partial^{2} c_{b}}{\partial x^{2}}-\xi\left(c_{b}-\left.c_{p}\right|_{\rho=1}\right) \\
\epsilon_{p} \frac{\partial c_{p}}{\partial \tau}+\left(1-\epsilon_{p}\right) a \frac{\partial q_{p}}{\partial \tau}=\frac{1}{\rho^{2}} \frac{\partial}{\partial r}\left(\rho^{2}\left[\epsilon_{p} \eta_{p} \frac{\partial c_{p}}{\partial \rho}+\left(1-\epsilon_{p}\right) a \eta_{s} \frac{\partial q_{p}}{\partial r}\right]\right)
\end{gathered}
$$

and

$$
\frac{\partial q_{p}}{\partial \tau}=k_{d}\left(c_{p}-q_{p}\right)
$$

The Eqs. (7)-(9) are also subjected to the initial and boundary conditions.

The initial conditions for an initially regenerated column are given as

$$
c_{b}(0, x)=0, \quad c_{p}(0, x, \gamma)=0, \quad q_{p}(0, x)=0, \quad \forall x, \gamma \in(0,1)
$$

For Eq. (8), the following boundary conditions at $\rho=0$ and $\rho=1$ are assumed:

$$
\left.\frac{\partial c_{p}}{\partial \rho}\right|_{\rho=0}=0, \quad \frac{\epsilon_{p}}{B i_{p}} \frac{\partial c_{p}}{\partial \rho}+\left.\frac{1-\epsilon_{p}}{B i_{s}} a \frac{\partial q_{p}}{\partial \rho}\right|_{\rho=1}=c_{b}-\left.c_{p}\right|_{\rho=1} .
$$

Appropriate inlet and outlet BCs are required for Eqs. (7). The following two sets of BCs are considered.

\section{Boundary conditions of type I: Dirichlet inlet BCs}

As a first choice, the simpler Dirichlet boundary conditions could be applied at the column inlet

$$
\left.c_{b}\right|_{x=0}=\left\{\begin{array}{lr}
1, & \text { if } 0<\tau \leq \tau_{\text {inj }} \\
0, & \tau>\tau_{\text {inj }}
\end{array}\right.
$$

where $\tau_{\text {inj }}$ is the dimensionless time of injection. The following Neumann boundary condition is applied at the outlet of a column of hypothetically infinite length, $x=\infty$

$$
\frac{\partial c_{b}(\tau, \infty)}{\partial x}=0
$$

For sufficiently small dispersion coefficient, for example $D_{b} \leq 10^{-5} \mathrm{~m}^{2} / \mathrm{s}$, this Dirichlet inlet boundary condition is well applicable. 


\section{Boundary conditions of type II: Danckwerts (or Robin) type inlet BCs}

Alternatively, the Danckwerts (or Robin) type boundary condition could be applied at the column inlet (e.g. Danckwerts (1953))

$$
-\frac{1}{P e} \frac{\partial c_{b}}{\partial x}+\left.c_{b}\right|_{x=0}=\left\{\begin{array}{rrr}
1, & \text { if } 0<\tau \leq \tau_{\mathrm{inj}}, \\
0, & \tau>\tau_{\mathrm{inj}} .
\end{array}\right.
$$

At the outlet of the column of finite length (i.e. at $x=1$ ), the following Neumann outflow boundary condition is applied:

$$
\frac{\partial c_{b}(1, \tau)}{\partial x}=0
$$

\section{Analytical solutions}

In this section, semi-analytical solutions of the linear GRM are presented for Dirichlet (Eq. (12a)) and Danckwerts (Eq. (13a)) inlet boundary conditions. The current model can conveniently be solved by means of Laplace transformation. The Laplace transformation is defined as

$$
\bar{c}(s, x)=\int_{0}^{\infty} \mathrm{e}^{-\mathrm{s} \tau}(\tau, x) \mathrm{d} \tau, \quad \tau \geq 0 .
$$

By applying the above Laplace definition on Eq. (7), we obtain

$$
s \overline{c_{b}}=\frac{1}{P e} \frac{\partial^{2} \overline{c_{b}}}{d x^{2}}-\frac{\partial \overline{c_{b}}}{d x}-\xi\left(\overline{c_{b}}-\left.\overline{c_{p}}\right|_{\rho=1}\right) .
$$

The Laplace transformation of Eqs. (8) is expressed as

$$
\epsilon_{p} s \overline{c_{p}}+\left(1-\epsilon_{p}\right) a s \overline{q_{p}}=\frac{1}{\rho^{2}} \frac{\partial}{\partial \rho}\left(\rho^{2}\left[\epsilon_{p} \eta_{p} \frac{\partial \overline{c_{p}}}{\partial \rho}+\left(1-\epsilon_{p}\right) a \eta_{s} \frac{\partial \overline{q_{p}}}{\partial r}\right]\right) .
$$

Moreover, the Laplace transformation of Eqs. (9) is given as

$$
\bar{q}=\frac{k_{d} \overline{c_{p}}}{s+k_{d}} \text {. }
$$

On using Eq. (16) in (15), we obtain

$$
\frac{\partial^{2} \overline{c_{p}}}{\partial \rho^{2}}+\frac{2}{\rho} \frac{\partial \overline{c_{p}}}{\partial \rho}=A(s) \overline{c_{p}},
$$


where

$$
A(s)=s \frac{\epsilon_{p}+\left(1-\epsilon_{p}\right) \frac{a k_{d}}{s k_{d}}}{\epsilon_{p} \eta_{p}+\left(1-\epsilon_{p}\right) \eta_{s} \frac{a k_{d}}{s+k_{d}}} .
$$

The general solution of (17) is given as

$$
\bar{c}_{p}(s, x)=\frac{1}{\rho}\left[k_{1} \cosh \sqrt{A(s)} \rho+k_{2} \sinh \sqrt{A(s)} \rho\right] .
$$

Using the Laplace transformation of the outlet boundary condition (BC) in Eq. (11), i.e. $\left.\frac{\partial \bar{c}_{p}}{\partial \rho}\right|_{\rho=0}$, Eq. (19) gives $k_{1}=0$. Thus, we obtain

$$
\bar{c}_{p}(s, x)=\frac{k_{2}}{\rho} \sinh \sqrt{A(s)} \rho .
$$

Now, taking the Laplace transformation of the inlet BC in Eq. (11), we obtain

$$
\left.\frac{1}{B(s)} \frac{\partial \overline{c_{p}}}{\partial \rho}\right|_{\rho=1}=\overline{c_{p}}-\left.\overline{c_{p}}\right|_{\rho=1}
$$

where

$$
B(s)=\frac{B i_{p} B i_{s}}{B i_{s} \epsilon_{p}+\left(1-\epsilon_{p}\right) B i_{p} \frac{a k_{d}}{s+k_{d}}} .
$$

On using Eq. (20) in Eq. (21), we obtain

$$
k_{2}=\frac{B(s) \bar{c}_{b}}{(B(s)-1) \sinh (\sqrt{A(s)})+\sqrt{A(s)} \cosh (\sqrt{A(s)})} .
$$

Thus, Eq. (20) at $\rho=1$ gives

$$
\left.\bar{c}_{p}(s, x)\right|_{\rho=1}=\frac{B(s)}{B(s)-1+\sqrt{A(s)} \operatorname{coth}(\sqrt{A(s)})} \bar{c}_{b}=f(s) \bar{c}_{b},
$$

where

$$
f(s)=\frac{B(s)}{B(s)-1+\sqrt{A(s)} \operatorname{coth}(\sqrt{A(s)})} .
$$

On using Eq. (24) in Eq. (14), we get

$$
\frac{\partial^{2} \bar{c}_{b}}{\partial x^{2}}-P e \frac{\partial \bar{c}_{b}}{\partial x}-\phi(s) P e \bar{c}_{b}=0,
$$


where

$$
\phi(s)=s+\xi(1-f(s))
$$

The general solution of Eq. (26) is given as

$$
\bar{c}_{b}(s, x)=A_{1} e^{\lambda_{1} x}+B_{1} e^{\lambda_{2} x},
$$

where

$$
\lambda_{1,2}(s)=\frac{P e}{2}\left(1 \mp \sqrt{1+\frac{4 \phi(s)}{P e}}\right) .
$$

The integration constants $A_{1}$ and $B_{1}$ can be obtained by using BCs either in Eqs. (12a) and (12b) or in Eqs. (13a) and (13b).

\section{Boundary conditions of type I: Dirichlet BCs}

Here, we consider the boundary conditions given by Eqs. (12a) and (12b). Their Laplace transformations are given as

$$
\overline{c_{b}}(s, 0)=\frac{\left(1-e^{-s \tau_{\mathrm{inj}}}\right)}{s}, \quad \frac{d \overline{c_{b}}}{d x}(s, \infty)=0 .
$$

Using Eq. (30) in Eq. (28), the values of $A_{1}$ and $B_{1}$ come out to be

$$
A_{1}=\frac{\left(1-e^{-s \tau_{\mathrm{inj}}}\right)}{s}, \quad B_{1}=0 .
$$

On plugging these values of $A_{1}$ and $B_{1}$ in Eq. (28), we get

$$
\overline{c_{b}}(s, x)=\frac{\left(1-e^{-s \tau_{\mathrm{inj}}}\right)}{s} e^{\lambda_{1} x},
$$

where, $\lambda_{1}(s)$ is given by Eq. (29) for the upper sign. When $\tau_{\text {inj }} \rightarrow \infty$, the injection causes a complete breakthrough curve. For this case, the solution in Eq. (32) reduces to the following form

$$
\overline{c_{b}}(s, x)=\frac{1}{s} e^{\lambda_{1} x}
$$




\section{Boundary conditions of type II: Danckwerts (or Robin) type BCs}

The Laplace transformation of the inlet Danckwerts BC in Eq. (13a) gives

$$
\bar{c}_{b}(s, 0)=\frac{1}{s}\left(1-e^{-s \tau_{\mathrm{inj}}}\right)+\left.\frac{1}{P e} \frac{d \bar{c}_{b}}{d x}\right|_{x=0} .
$$

Similarly, the Laplace transformation of the outlet BC in Eq. (13b) gives

$$
\left.\frac{d \overline{c_{b}}(s, x)}{d x}\right|_{x=1}=0 .
$$

Using Eqs. (34) and (35) in Eq. (28), the values of $A_{1}$ and $B_{1}$ are obtained as

$$
\begin{gathered}
A_{1}=\frac{\left(1-e^{-s \tau_{\mathrm{inj}}}\right)}{s} \frac{\lambda_{2} e^{\lambda_{2}}}{\left(1-\frac{\lambda_{1}}{P e}\right) \lambda_{2} e^{\lambda_{2}}-\left(1-\frac{\lambda_{2}}{P e}\right) \lambda_{1} e^{\lambda_{1}}}, \\
B_{1}=-\frac{\left(1-e^{-s \tau_{\mathrm{inj}}}\right)}{s} \frac{\lambda_{1} e^{\lambda_{1}}}{\left(1-\frac{\lambda_{1}}{P e}\right) \lambda_{2} e^{\lambda_{2}}-\left(1-\frac{\lambda_{2}}{P e}\right) \lambda_{1} e^{\lambda_{1}}} .
\end{gathered}
$$

At $x=1$, the complete solution has the following simple form:

$$
\bar{c}_{b}(s, x)=\frac{\left(1-e^{-s \tau_{\mathrm{inj}}}\right)}{s} \frac{\lambda_{2} e^{\lambda_{1}+\lambda_{2}}-\lambda_{1} e^{\lambda_{1}+\lambda_{2}}}{\left(1-\frac{\lambda_{1}}{P e}\right) \lambda_{2} e^{\lambda_{2}}-\left(1-\frac{\lambda_{2}}{P e}\right) \lambda_{1} e^{\lambda_{1}}} .
$$

When $\tau_{\text {inj }} \rightarrow \infty$, the injection again causes a breakthrough curve. In such a situation, Eq. (38) reduces to

$$
\bar{c}_{b}(s, x)=\frac{1}{s} \frac{\lambda_{2} e^{\lambda_{1}+\lambda_{2}}-\lambda_{1} e^{\lambda_{1}+\lambda_{2}}}{\left(1-\frac{\lambda_{1}}{P e}\right) \lambda_{2} e^{\lambda_{2}}-\left(1-\frac{\lambda_{2}}{P e}\right) \lambda_{1} e^{\lambda_{1}}} .
$$

This completes the discussion of analytical solutions for the single component linear GRM in the Laplace domain.

There is no possibility to analytically transform back the Laplace domain solution in the actual time domain. The numerical Laplace inversion can be applied to obtain a discrete solution in time. In this technique, the integral of inverse Laplace transformation is approximated by Fourier series, see for example Rice et al. (1995). However, this solution is not helpful for the parameters estimation from experimental data. For that purpose, temporal moments are derived in the next section. 


\section{Moment analysis}

Moment analysis is an effective technique for extracting important information about the retention and mass transfer processes in chromatographic columns, see e.g. Kucera (1965); Schneider and Smith (1968); Ruthven (1984); Guiochon et al. (2006); Miyabe et al. (2007, 2009). The Laplace domain solutions can be used to obtain moments. In this section, the temporal moments up to fourth order for the GRM are derived. The following moment generating property of the Laplace transform is exploited (e.g. Van der Laan (1958))

$$
\mu_{0}=\lim _{s \rightarrow 0}(\bar{c}(s, x=1)), \quad \mu_{n}=(-1)^{n} \frac{1}{\mu_{0}} \lim _{s \rightarrow 0} \frac{d^{n}(\bar{c}(s, x=1))}{d s^{n}}, \quad n=1,2,3, \cdots .
$$

The second, third and fourth central moments can be obtained as

$$
\mu_{2}^{\prime}=\mu_{2}-\mu_{1}^{2}, \quad \mu_{3}^{\prime}=\mu_{3}-3 \mu_{1} \mu_{2}+2 \mu_{1}^{3}, \quad \mu_{4}^{\prime}=\mu_{4}-4 \mu_{1} \mu_{3}+6 \mu_{1}^{2} \mu_{2}-3 \mu_{1}^{4} .
$$

It is well known that the zeroth moment $\mu_{0}$ gives the mass (or peak area), the first moment $\mu_{1}$ corresponds to the retention time $\tau_{R}$. The effects of longitudinal diffusion are not significant with respect to retention time or first moment. The second central moment $\mu_{2}^{\prime}$, i.e. the variance of the elution profile, provides information about the rates of the mass transfer processes in the column. The third central moment $\mu_{3}^{\prime}$ quantifies the front asymmetries. Lastly, the fourth central moment $\mu_{4}^{\prime}$ measures the kurtosis. The complete derivations of moments are presented using the considered two types of boundary conditions.

\section{Boundary conditions of type I: Dirichlet BCs}

The final solution for Dirichlet BCs (c.f. Eq. (30)) is given by Eq. (33). The moments of this solution are given as follows.

Zeroth moment: The zeroth moment for rectangular profiles is given as

$$
\mu_{0}=\lim _{s \rightarrow 0}\left(\overline{c_{b}}(s, x=1)\right)=\tau_{\text {inj }} .
$$

The zeroth moment for continuous breakthrough curves is simply $\mu_{0}=1$.

First moment: The first temporal moment for rectangular profiles is given as (c.f. Eq. $(40))$

$$
\mu_{1}=\frac{\tau_{\text {inj }}}{2}+1+a^{*} F
$$


where

$$
a^{*}=\epsilon_{p}+\left(1-\epsilon_{p}\right) a .
$$

For continuous breakthrough curves, the first term on the right hand side of Eq. (43) in is zero, i.e.

$$
\mu_{1}=1+a^{*} F
$$

Second central moment: For a rectangular profile it is given as

$$
\mu_{2}^{\prime}=\frac{\tau_{\mathrm{inj}}^{2}}{12}+\frac{2}{P e}\left(1+a^{*} F\right)^{2}+F\left(\frac{2 F a^{* 2}}{\xi}+\frac{2 a^{* 2}}{15 \eta}\right)+2 \frac{F\left(a^{*}-\epsilon_{p}\right)}{k_{d}},
$$

where

$$
\eta=L \frac{\epsilon_{p} D_{p}+\left(1-\epsilon_{p}\right) a D_{s}}{u R_{p}^{2}} .
$$

For breakthrough curves the first term on the right hand side of Eq. (46) is zero, i.e.

$$
\mu_{2}^{\prime}=\frac{2}{P e}\left(1+a^{*} F\right)^{2}+F\left(\frac{2 F a^{* 2}}{\xi}+\frac{2 a^{* 2}}{15 \eta}\right)+2 \frac{F\left(a^{*}-\epsilon_{p}\right)}{k_{d}} .
$$

The first and second central moments can be used to obtain a relation for the flowrate dependent Height Equivalent to Theoretical Plate (HETP) number. To derive the classical HETP-curve, let us consider for the sake of simplicity a Dirac injection, i.e. $\tau_{\text {inj }}=0$. Then, Eqs. (43), and (46) gives (e.g. Guiochon et al. (2006); Van Deemter et al. (1956))

$$
\operatorname{HETP}_{\text {Diric }}(u)=\frac{L \mu_{2}^{\prime}}{\mu_{1}^{2}}=\frac{2 D_{b}}{u}+\frac{2 L F}{u\left(1+a^{*} F\right)^{2}}\left[\frac{F a^{* 2}}{\xi}+\frac{a^{* 2}}{15 \eta}+\frac{\left(a^{*}-\epsilon_{p}\right)}{k_{d}}\right] u .
$$

In the above equation, the first term captures the band broadening by dispersion, the second term describes the contribution of external mass transfer, and the third term provides the contribution of diffusive migration of sample molecules inside the stationary phase, and the last term represents the contribution of desorption rate constant. The first term is frequently splitted using the following simplifying expression (e.g. Ruthven (1984); Guiochon et al. (2006))

$$
D_{b}=\gamma_{1} 2 R_{p} u+\gamma_{2} D_{\mathrm{mol}}
$$


Here, $D_{\text {mol }}$ is the molecular diffusivity of the solute in the solvent, while $\gamma_{1}$ and $\gamma_{2}$ represent weight factors for the relative impact of the particle size/flow rate dependent and the molecular diffusion based contributions to band broadening. Thus, Eq. (49) can be rewritten as

$$
\operatorname{HETP}_{\text {Diric }}(u)=4 \gamma_{1} R_{p}+\frac{2 \gamma_{2} D_{\text {mol }}}{u}+C_{\text {core }} u=A+\frac{B}{u}+C u,
$$

where

$$
A=4 \gamma_{1} R_{p}, \quad B=2 \gamma_{2} D_{\mathrm{mol}}, \quad C=\frac{2 L F}{u\left(1+a^{*} F\right)^{2}}\left[\frac{F a^{* 2}}{\xi}+\frac{a^{* 2}}{15 \eta}+\frac{\left(a^{*}-\epsilon_{p}\right)}{k_{d}}\right] .
$$

Third central moment: The third central moment is calculated as (c.f. Eq. (40))

$$
\begin{aligned}
\mu_{3}^{\prime}= & \frac{12}{P e^{2}}\left(1+a^{*} F\right)^{3}+\frac{6\left(1+a^{*} F\right) F}{P e}\left(\frac{2 F a^{* 2}}{\xi}+\frac{2 a^{* 2}}{15 \eta}\right)+F a^{* 3}\left(\frac{4}{105 \eta^{2}}+\frac{4 F}{5 \xi \eta}+\frac{6 F^{2}}{\xi^{2}}\right) \\
& +12 \frac{a F\left(1-\epsilon_{p}\right)}{k_{d}}\left[\frac{1+a^{*} F}{P e}+\frac{a^{*} F}{\xi}+\frac{a^{*}}{15 \eta}+\frac{1}{2 k_{d}}\right] .
\end{aligned}
$$

Moreover, $\mu_{3}^{\prime}$ is the same for rectangular and continuous breakthrough curves.

The second and third central moments can be used to calculate the skewness that measures the degree of asymmetry of elution profiles. It can be evaluated as

$$
\delta_{\text {skew }}=\frac{\mu_{3}^{\prime}}{\left(\mu_{2}^{\prime}\right)^{3 / 2}} .
$$

This relation is useful to quantify deviations from Gaussian peak shapes. As Eq. (54) indicates, chromatographic peaks corresponding to GRM have some degree of asymmetry. Thus, their front and rare parts have not the same shape. 
Fourth central moment: The fourth temporal moment is obtained as (c.f. Eq. (40))

$$
\begin{aligned}
\mu_{4}= & \frac{12\left(1+a^{*} F\right)^{4}}{P e^{3}}(P e+10)+\frac{24\left(1+a^{*} F\right)^{2} F}{P e^{2}}\left(\frac{F a^{* 2}}{\xi}+\frac{a^{* 2}}{15 \eta}\right)(6+P e) \\
& +\frac{48 F^{3} a^{* 3}}{\xi^{2} P e}+\frac{48 F^{4} a^{* 4}}{\xi^{2} P e}+\frac{48 F^{3} a^{* 4}}{5 \xi \eta P e}+\frac{32 F^{2} a^{* 3}}{5 \xi \eta P e}+\frac{32 F a^{* 3}}{105 \eta^{2} P e}+\frac{72 F^{2} a^{* 4}}{175 \eta^{2} P e} \\
& +\frac{12 F^{4} a^{* 4}}{\xi^{2}}+\frac{24 F^{4} a^{* 4}}{\xi^{3}}+\frac{24 F^{3} a^{* 4}}{5 \eta \xi^{2}}+\frac{72 F^{2} a^{* 4}}{175 \xi \eta^{2}}+\frac{8 F^{3} a^{* 4}}{5 \xi \eta}+\frac{4 a F^{2} a^{* 4}}{75 \eta^{2}} \\
& \frac{-24 F\left(\epsilon_{p}-1\right)+12\left(F\left(\epsilon_{p}-1\right)\right)^{2}+\frac{8 F\left(\left(\epsilon_{p}-1\right)\right)^{2}}{B i \eta}+\frac{8 F\left(\left(\epsilon_{p}-1\right)\right)^{2}}{5 \eta}}{k_{d}^{3}} \\
& \frac{-16 a F a^{*}\left(\epsilon_{p}-1\right)}{B_{i} \eta k_{d}^{2}}-\frac{16 a F a^{*}\left(\epsilon_{p}-1\right)}{5 \eta k_{d}^{2}}-\frac{8 a F^{2} a^{* 2}\left(\epsilon_{p}-1\right)}{5 \eta k_{d}}-\frac{16 a F a^{* 2}\left(\epsilon_{p}-1\right)}{5 B i \eta^{2} k_{d}} \\
& -\frac{8 a F^{2} a^{* 2}\left(\epsilon_{p}-1\right)}{B i \eta k_{d}}-\frac{8 a F a^{* 2}\left(\epsilon_{p}-1\right)}{k_{d} B i^{2} \eta^{2}}-\frac{16 a F a^{* 2}\left(\epsilon_{p}-1\right)}{35 \eta k_{d}} \\
& +\frac{\tau_{\text {inj }}^{4}}{80}+\tau_{\mathrm{inj}}^{2} \frac{\left(1+a^{*} F\right)^{2}}{P e}+\tau_{\mathrm{inj}}^{2} F\left[\frac{F a^{* 2}}{\xi}+\frac{a^{* 2}}{15 \eta}\right]
\end{aligned}
$$

where

$$
B i=\frac{B i_{p} B i_{s}}{B i_{s} \epsilon_{p}+\left(1-\epsilon_{p}\right) a B i_{p}} .
$$

For breakthrough curves all terms in $\mu_{4}^{\prime}$ containing $\tau_{\text {inj }}$ are zero. The fourth central moment can be utilized to calculate kurtosis which measures the profiles peakedness or flatness relative to a normal distribution. In general, the kurtosis is a descriptor of the shape of a probability distribution. It is instructive to use an adjusted version of Pearson's kurtosis, the excess kurtosis, (see DeCarlo (1997)). The excess kurtosis compares the shape of a given distribution to that of the normal distribution. Distributions with negative or positive excess kurtosis are called platykurtic distributions or leptokurtic distributions, respectively. The following definition quantifies the excess kurtosis

$$
\gamma=\frac{\mu_{4}^{\prime}}{\left(\mu_{2}^{\prime}\right)^{2}}-3 .
$$

A high kurtosis distribution has a sharper peak and a broader tails than the normal distribution, while a low kurtosis distribution has a more rounded peak and thinner tails. Distributions with zero excess kurtosis, as the normal distribution, are called mesokurtic. 
The above moments were presented in terms of the dimensionless time coordinate $\tau$. However, they can be easily expressed in term of the actual time coordinate $t:=L \tau / u$ as follows:

$$
\mu_{0}(t)=\frac{L}{u} \mu_{0}(\tau), \quad \mu_{i}(t)=\left(\frac{L}{u}\right)^{i} \mu_{i}(\tau), \quad i=1,2,3 .
$$

\section{Boundary conditions of type II: Danckwerts (or Robin) type BCs}

In this case, the BCs given by Eqs. (34) and (35) are considered. The final solution is given by Eq. (38). The moments of this solution are given below.

Zeroth moment: The zeroth moment for rectangular profiles is given as

$$
\mu_{0}=\tau_{\text {inj }}
$$

First moment: The first temporal moment using Eq. (40) is calculated as

$$
\mu_{1}=\frac{\tau_{\text {inj }}}{2}+1+a^{*} F
$$

Second central moment: It is given as

$$
\mu_{2}^{\prime}=\frac{\tau_{\text {inj }}^{2}}{12}+\frac{2\left(1+a^{*} F\right)^{2}}{P e}\left[1+\frac{1}{P e}\left(e^{-P e}-1\right)\right]+F\left[\frac{2 F a^{* 2}}{\xi}+\frac{2 a^{* 2}}{15 \eta}\right]+\frac{2 F\left(a^{*}-\epsilon_{p}\right)}{k_{d}},
$$

In this case the expression in Eqs. (60) and (61) can be used to calculate HETP-curve for the Danckwerts BCs. Thus, we finally obtain

$$
\operatorname{HETP}_{\text {Danck }}(u)=\frac{L \mu_{2}^{\prime}}{\mu_{1}^{2}}=4 \gamma_{1} R_{p}+\frac{2 \gamma_{2} D_{\mathrm{mol}}}{u}+C u=\tilde{A}+\frac{\tilde{B}}{u}+C u,
$$

where $C$ is given by Eq. (52) and $\tilde{A}$ and $\tilde{B}$ are given as

$$
\tilde{A}=4 \gamma_{1} R_{p}\left[1+\frac{1}{P e}\left(e^{-P e}-1\right)\right], \quad \tilde{B}=2 \gamma_{2} D_{\text {mol }}\left[1+\frac{1}{P e}\left(e^{-P e}-1\right)\right] .
$$

Third central moment: It is expressed as

$$
\begin{aligned}
\mu_{3}^{\prime}= & \frac{12}{P e^{2}}\left(1+a^{*} F\right)^{3}\left[\left(1+\frac{2}{P e}\right) e^{-P e}+\left(1-\frac{2}{P e}\right)\right] \\
& +\frac{6\left(1+a^{*} F\right) F}{P e}\left(\frac{2 F a^{* 2}}{\xi}+\frac{2 a^{* 2}}{15 \eta}\right)\left[\frac{1}{P e}\left(e^{-P e}-1\right)+1\right]+F a^{* 3}\left(\frac{4}{105 \eta^{2}}+\frac{4 F}{5 \xi \eta}+\frac{6 F^{2}}{\xi^{2}}\right) \\
& +12 \frac{a F\left(1-\epsilon_{p}\right)}{k_{d}}\left(\frac{1+a^{*} F}{P e}\left[\frac{1}{P e}\left(e^{-P e}-1\right)+1\right]+\frac{a^{*} F}{\xi}+\frac{a^{*}}{15 \eta}+\frac{1}{2 k_{d}}\right) .
\end{aligned}
$$

The expression of $\mu_{4}^{\prime}$ was very large for Danckwarts BCs. Therefore, only plots of that moment are presented. 


\section{Numerical test problems}

In this section, at first the effect of $K_{d}$, i.e. the desorption rate, is analyzed. Then, the effects of Pe, $\eta$ (c.f. Eq. (47)) and Bi (c.f. Eq. (56)), characterizing the axial dispersion, intraparticle diffusion resistance and film mass transfer resistance, on the concentration profiles and moments are investigated. Moreover, effects of external mass transfer coefficient $k_{\text {ext }}$, as well as pore and surface diffusivities $\left(D_{p}\right.$ and $\left.D_{s}\right)$ on elution profiles are analyzed. A second-order accurate finite volume scheme (FVS) is chosen to numerically approximate the model equations for verifying the correctness of derived analytical results, see Javeed et al. (2011). All parameters used in the test problems are given in Table 1.

Figures 1a shows a comparison of the concentration profiles for different values of the $K_{d}$. As $K_{d}$ increases from 0.1 to $10^{4} \mathrm{sec}^{-1}$, the elution profiles become narrow, tall and symmetric. Thus, the column has better efficiency for large value of $K_{d}$ and is more useful for purification and separation of multi-component mixtures. Beyond $K_{d}=10^{4}$, the remaining dispersion is due to the other kinetic effects being active in the GRM and the adsorption equilibrium is permanently fulfilled. Since, there are typically no precise data regarding the system specific rate of adsorption and desorption available, a more general evaluation of the border value for $K_{d}$ and an independent validation are not easy and out of the scope of our paper. Here, we essentially illustrate just the possibility to quantify the consequences of the effect. Figures $1 \mathrm{~b}$ sows that analytical and numerical solutions of the finite volume scheme (FVS) are in good agreement with each other for all values of $K_{d}$. This is an indication of the accuracy of proposed numerical algorithm and the derived analytical solutions.

The effects of model parameters $P e, B i, \eta$, and the considered two sets of BCs on the elution curves are shown in Figure 2. It is evident that if axial dispersion or film mass transfer resistance is important, the peak becomes wider and time corresponding to the peak maximum is slightly lower. The effect of $K_{d}$ is same as in Figure 1. For small values of $B_{i}$ and $\eta$, the diffusion rate is very slow and fluid retention time is large. The breakthrough time becomes shorter due to the limitation of the intraparticle diffusion resistance. For low 
efficient columns, i.e. small $P e$ numbers (low flowrates, short columns, large back-mixing effects), the selection of boundary conditions influences the results and the more realistic Danckwerts BC and the corresponding solutions should be applied (c.f. Figure 2d). If $P e$ is very large Eq. (63) converges into Eq. (52).

The elution profiles for different values of external mass transfer coefficient are shown in Figure 3a. Here, $K_{d}=10 \mathrm{sec}^{-1}$ is fixed. It is interesting that the band broadening decreases significantly when $k_{\text {ext }}$ increases from $10^{-6} \mathrm{~cm} / \mathrm{sec}$ to $10^{-4} \mathrm{~cm} / \mathrm{sec}$, but when $k_{\text {ext }}$ increases to $10^{-1} \mathrm{~cm} / \mathrm{sec}$, the decrease of band broadening is not remarkable and for $10^{-4} \mathrm{~cm} / \mathrm{sec}$ and $10^{-1} \mathrm{~cm} / \mathrm{sec}$ the calculated profiles can hardly be distinguished. The result suggests us that the effect of the external mass transfer resistance can be neglected when the value of $k_{\text {ext }}$ is more than $10^{-1} \mathrm{~cm} / \mathrm{sec}$. The effect of $D_{p}=D_{s}$ on the eluted band profile is shown in Figure 3a for $K_{d}=10 \mathrm{sec}^{-1}$. The figure indicates that the band broadening is sensitive to the values of $D_{p}=D_{s}$. It can be seen that on increasing $D_{p}=D_{s}$, the band broadening decreases.

Figure 4 displays the first four dimensional temporal moments (c.f. Eq. (58)) which endorse all observations of Figure 1. Eqs. (43) and (44) indicate that retention time of the solute molecules is not correlated with $K_{d}$. Thus, as required, the retention time (i.e. $\left.\mu_{1}\right)$ was not effected on changing the value of $K_{d}$. However, the variance $\mu_{2}^{\prime}$, the third central moment $\mu_{3}^{\prime}$, and the fourth central moment $\mu_{4}^{\prime}$ were reduced in different ways on increasing the value of $K_{d}$. Obviously, the results are very similar for $K_{d}=10^{3}$ and $10^{4}$. Thus, for values of $K_{d}$ larger then $10^{4}$ the adsorption equilibrium is more or less established and the reduced GRM can be used.

Figure 5 displays the skewness (c.f. Eq. (54)) and excess kurtosis (c.f. Eq. (57)) as functions of velocity for different values of $K_{d}$. It can be observed that the concentration profile is more asymmetrical for small values of velocity $u$ and $K_{d}$ (see also Figures 1 ). Moreover, the excess kurtosis or flatness of the profile increases on increasing $K_{d}$. Once again, it is visible that for $K_{d}=10^{4}$ the equilibrium is reached.

Figure 6a shows the plots of plate heights HETP (c.f. Eqs. (51) and (52)) as functions of the velocity $u$. The illustrating calculations were done for the Danckwerts BC and 
different values of $K_{d}$. It can be seen that HETP-values become smaller with an increase in $K_{d}$. Figure $6 \mathrm{~b}$ illustrates differences in the HETP of considered two types of boundary conditions. HETP is smaller for the Dankweets BCs due to the joint effects of the different inlet and out boundary conditions (Eqs. (12a) and (12b) vs Eqs. (13a) and (13b)).

\section{Conclusion}

Semi-analytical solutions and temporal moments of the GRM were derived considering dynamic linear sorption kinetics and two different sets of boundary conditions. The model equations were analytically solved in the Laplace domain and numerical Laplace inversion was applied to get back solutions in the actual time domain. Analytical expression of the first four temporal moments were derived from solutions in the Laplace domain. The derived solutions and moments were used to analyze the effect of finite adsorption-desorption rates, axial dispersion, film mass transfer resistance and intraparticle diffusion resistance on the elution curves. In the parametric calculations carried out, using the solutions derived, emphasis was put on providing insight in the effect of finite desorption rate. The results showed that a small value of $K_{d}$ produces spreading in the elution profiles and the profiles become more asymmetric, causing reduction in the efficiency of the column. The analytical results were validated against the numerical results of second order finite volume scheme. Good agreements between analytically and numerically determined results verified the correctness of analytical solutions and accuracy of the suggested numerical scheme.

Antos, D., Kaczmarski, K., Wojciecha, P., Seidel-Morgenstern, A., 2003. Concentration dependence of lumped mass transfer coefficients: Linear versus non-linear chromatography and isocratic versus gradient operation. J. Chromatogr. A 1006, 61-76.

Carta, G., 1988. Exact analytical solution of a mathematical model for chromatographic operations. Chem. Eng. Sci. 43, 2877-2883.

Danckwerts, P.V., 1953. Continuous flow systems. Chem. Eng. Sci. 2, 1-9.

DeCarlo L.T., 1997. On the meaning and use of kurtosis. Psychological Methods 2, 292-307. 
Felinger, A., Guiochon, G., 2004. Comparison of the kinetic models of linear chromatography. Chromatogr. Suppl. 60, S175-S180.

Gengliang, Y., Zhide, H., 1996. Universal theoretical moment expressions for elution and frontal chromatography of pellicular ion exchange resins. Reactive and Functional Polymers $31,25-29$.

Guiochon, G., Felinger, A., Shirazi, D.G., Katti, A.M., 2006. Fundamentals of preparative and nonlinear chromatography, 2nd ed. ELsevier Academic press, New York.

Guiochon, G., Lin, B., 2003. Modeling for preparative chromatography, Academic Press.

Hougen, O.A., Marshal, W.R., 1947. Adsorption from a fluid stream flowing througha stationary granular bed, Chem. Eng. Prog. Trans. Sect. 3c, 197-208.

Javeed, S., Qamar, S., Seidel-Morgenstern, A., Warnecke, G., 2011. Efficient and accurate numerical simulation of nonlinear chromatographic processes. Comput. Chem. Eng. 35, 2294-2305.

Javeed, S., Qamar, S., Ashraf, W., Seidel-Morgenstern, A., Warnecke, G., 2013. Analysis and numerical investigation of two dynamic models for liquid chromatography. Chem. Eng. Sci. 90, 17-31.

Kitous, O., Abdi, N., Lounici, H., Grib, H., Drouiche, N., Benyoussef, E.H., Mameri, N., 2016. Modeling of the adsorption of metribuzin pesticide onto electro-activated granular carbon. Desalin. Water Treat. 57, 1865-1873.

Kubin, M., 1965. Beitrag zur Theorie der Chromatographie. Collet. Czechoslovak Chem. Commun. 30, 1104-1118.

Kubin, M., 1965. Beitrag zur theorie der chromatographie II. Einfluss der diffusion ausserhalb und der adsorption innerhalb des sorbens-korns. Collect. Czechoslovak Chem. Commun. 30, 2900-2907. 
Kucera, E., 1965. Contribution to the theory of chromatography: Linear non-equilibrium elution chromatography. J. Chromatogr. A 19, 237-248.

Lemlikchi, W., Drouiche, N., Belaicha, N., Oubagha, N., Baaziz, B., Mecherri, M.O., 2015. Kinetic study of the adsorption of textile dyes on synthetic hydroxyapatite in aqueous solution. Ind. Eng. Chem. 32, 233-237.

Lenhoff, A.M., 1987. Significance and estimation of chromatographic parameters. J. Chromatogr. A 384, 285-299.

Li, P., Xiu, G., Rodrigues, A.E., 2003. Analytical breakthrough curves for inert core adsorbent with sorption kinetics, AIChE J. 49, 2974-2979.

Li, P., Xiu, G., Rodrigues, A.E., 2004. Modeling breakthrough and elution curves in fixedbed of inert core adsorbents: analytical and approximate solutions. Chem. Eng. Sci. 59, 3091-3103.

Miyabe, K., Guiochon, G., 2003. Measurement of the parameters of the mass transfer kinetics in high performance liquid chromatography. J. separat. Sci. 26, 155-173.

Miyabe, K., 2007. Surface diffusion in reversed-phase liquid chromatography using silica gel stationary phases of different C1 and C18 ligand densities. J. Chromatogr. A 1167, 161-170.

Miyabe, K., 2009. Moment analysis of chromatographic behavior in reversed-phase liquid chromatography. J. separat. Sci. 32, 757-770.

Miyabe, K., 2014. Moment equations for chromatography based on Langmuir type reaction kinetics. J. Chromatogr. A 1356, 171-179.

Qamar, S., Abbasi, J.N., Javeed, S., Seidel-Morgenstern, A., 2014. Analytical solutions and moment analysis of general rate model for linear liquid chromatography. Chem. Eng. Sci. 107, 192-205. 
Qamar, S., Abbasi, J.N., Mehwish, A., 2015. Linear general rate model of chromatography for core-shell particles: Analytical solutions and moment analysis. Chem. Eng. Sci. 137, 352-363.

Rasmuson, A., 1985. Exact solution of a model for diffusion in particles and longitudinal dispersion in packed beds: numerical evaluation, AIChE J. 31, 518-519.

Rice, R.G., Do, D.D., 1995. Applied Mathematics and Modeling for Chemical Engineers, Wiley-Interscience, New York.

Rosen, J. B., 1952. Kinetics of a fixed bed system for solid diffusion into spherical particles, J. Chem. Phys. 20, 387-394.

Ruthven, D.M., 1984. Principles of adsorption and adsorption processes, WileyInterscience, New York.

Schmidt-Traub, H., Schulte, M., Seidel-Morgenstern, A., (2012). Preparative Chromatography, Wiley, Weinheim, Germany.

Schneider, P., Smith, J.M., 1968. Adsorption rate constants from chromatography. AIChE J. 14, 762-771.

Shams, K., Fayazbakhsh, A. (2014). Dynamics of reactive chromatographic columns of inertcore/hollow/film coated spherical packing: An analytical solution and applications. J. Chromatogr. A, 1370, 93-104.

Suzuki, M., 1973. Notes on Determining the Moments of the Impulse Response of the Basic Transformed Equations. J. Chem. Eng. Jpn 6, 540-543.

Thomas, H.C., 1947. Chromatography: a problem in kinetics. Ann. N.Y. Acad. Sci. 49, 161182.

Thomas, H.C., 1947. Solid diffusion in chromatography. J. Chem. Phys. 19, 1213-1233. 
Wolff, H.-J., Radeke, K.-H, Gelbin, D., 1980. Heat and mass transfer in packed beds-IV use of weighted moments to determine axial dispersion coefficient. Chem. Eng. Sci. 34, 101-107.

Wolff, H.-J., Radeke, K.-H, Gelbin, D., 1980. Weighted moments and the pore-diffusion model. Chem. Eng. Sci. 35, 1481-1485.

Van Deemter, J.J., Zuiderweg, F.J., Klinkenberg, A., 1956. Longitudinal diffusion and resistance to mass transfer as causes of nonideality in chromatography, Chem. Eng. Sci. 5, 271-289.

Van der Laan, Th., 1958. Letter to the Editors on Notes on the diffusion type model for the longitudinal mixing in flow. Chem. Eng. Sci. 7, 187-191 
Table 1: Parameters of the problems.

\begin{tabular}{|c|c|}
\hline Parameters & values \\
\hline Column length & $L=15 \mathrm{~cm}$ \\
Stationary phase particle radius & $R_{p}=5 \times 10^{-6} \mathrm{~cm}$ \\
External porosity & $\gamma_{1}=0.4$ \\
Geometrical constant & $\gamma_{2}=1.0$ \\
Geometrical constant & $\epsilon_{p}=0.333$ \\
Internal porosity & $u=0.4 \mathrm{~cm} / \mathrm{sec}$ \\
Interstitial velocity & $D_{b}=10^{-3} \mathrm{~cm}^{2} / \mathrm{sec}$ \\
Axial dispersion coefficient & $D_{\text {mol }}=10^{-5} \mathrm{~cm}^{2} / \mathrm{sec}$ \\
Molecular diffusivity & $D_{p}=10^{-6} \mathrm{~cm}^{2} / \mathrm{sec}$ \\
Pore diffusivity & $D_{s}=10^{-6} \mathrm{~cm}^{2} / \mathrm{sec}$ \\
Surface diffusivity & $a=2.5$ \\
Henry's constant & $K_{d}=0$ to $10^{4} \mathrm{sec}$ \\
Desorption rate constant & $k_{\text {ext }}=1.0 \mathrm{~cm}^{-1} \mathrm{sec}$ \\
External mass transfer coefficient & $c(0, z)=0 \mathrm{~g} / \mathrm{l}$ \\
Initial concentration & $c_{p}(0, z)=0 \mathrm{~g} / \mathrm{l}$ \\
Initial concentration & $c_{\text {inj }}=1.0 \mathrm{~g} / \mathrm{l}$ \\
Concentration at inlet & $t_{\text {inj }}=20 \mathrm{sec}$ \\
Injection time & \\
\hline
\end{tabular}



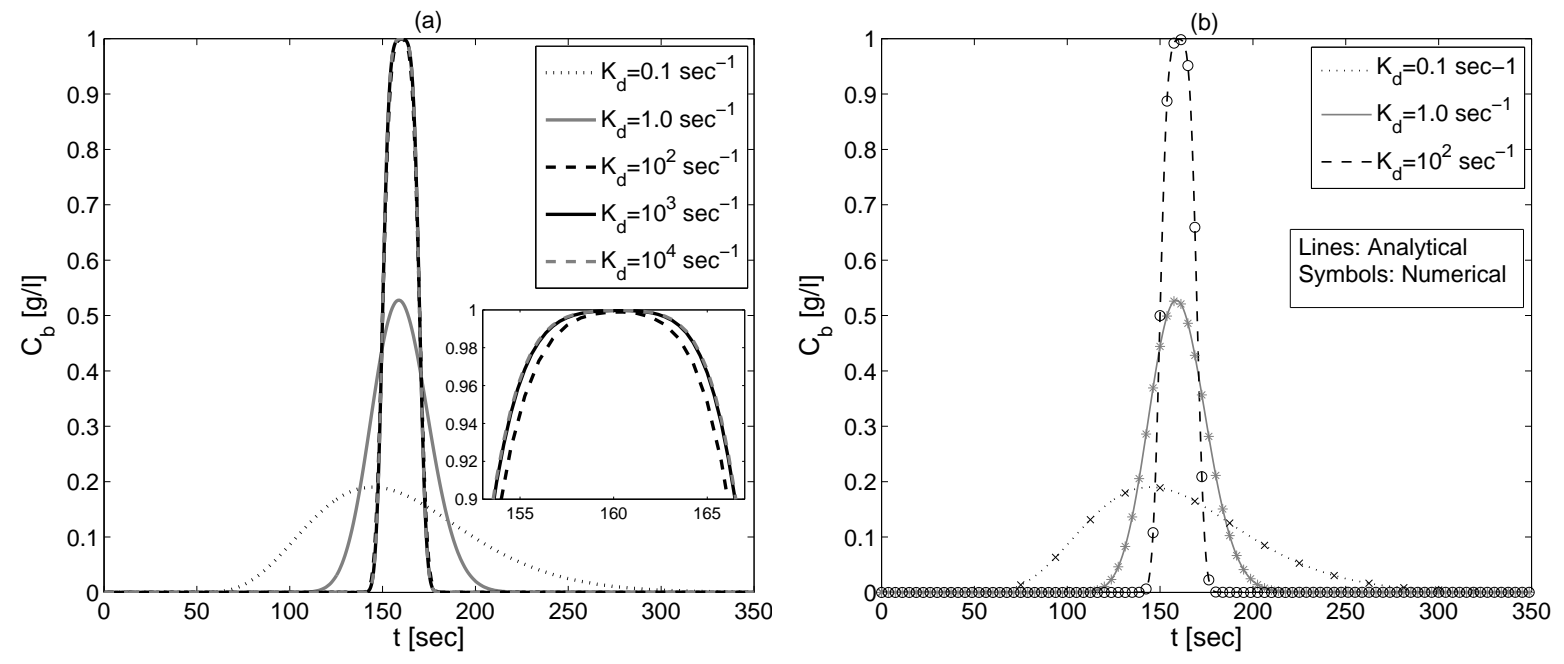

Figure 1: Effect of $K_{d}$ on the concentration profiles. All parameters are given in Table 1. 

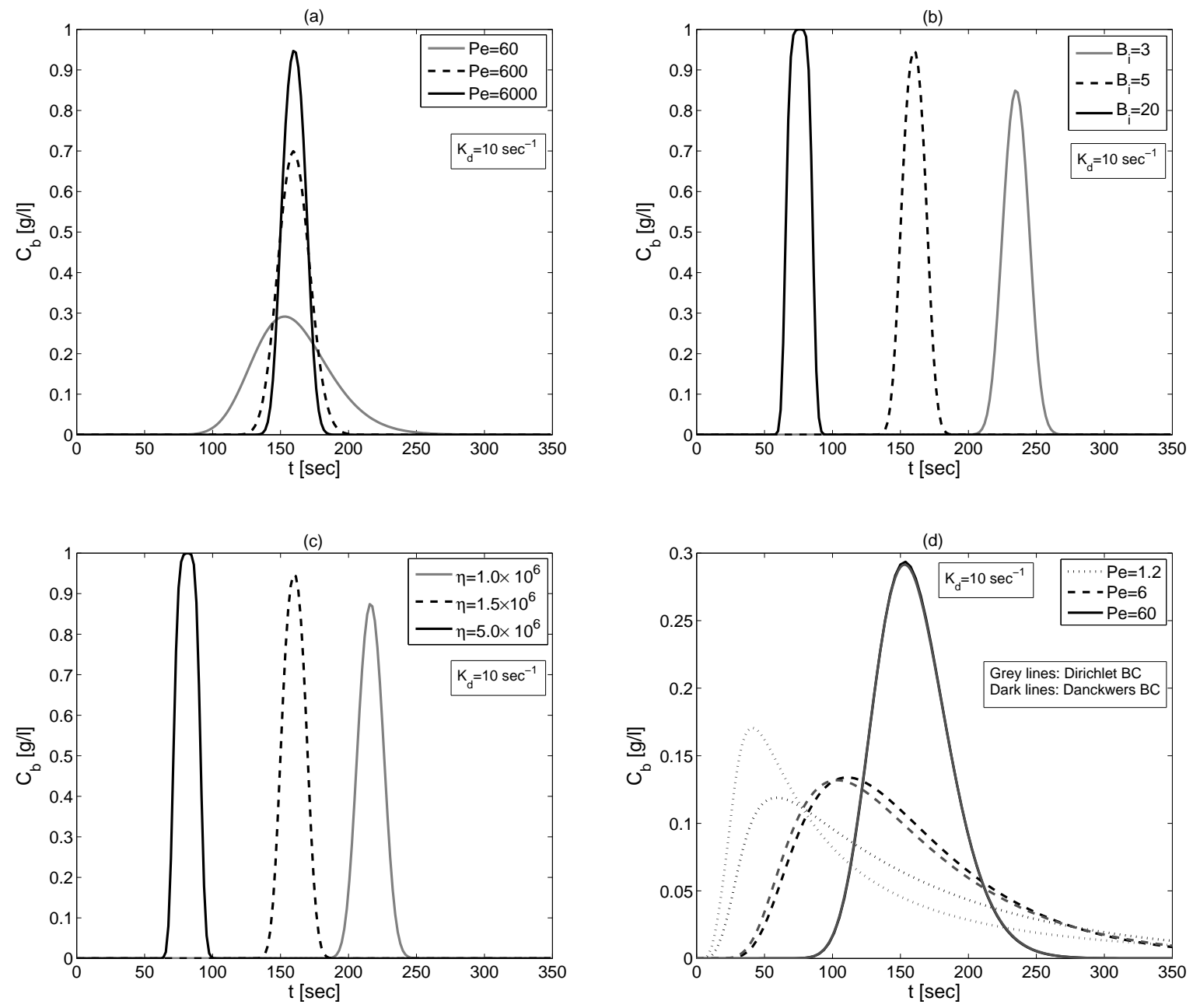

Figure 2: Effect of $P e, B_{i}$ and $\eta$, and BCs on the concentration profiles for fixed $K_{d}=10 \mathrm{sec}^{-1}$. Other parameters are given in Table 1. 

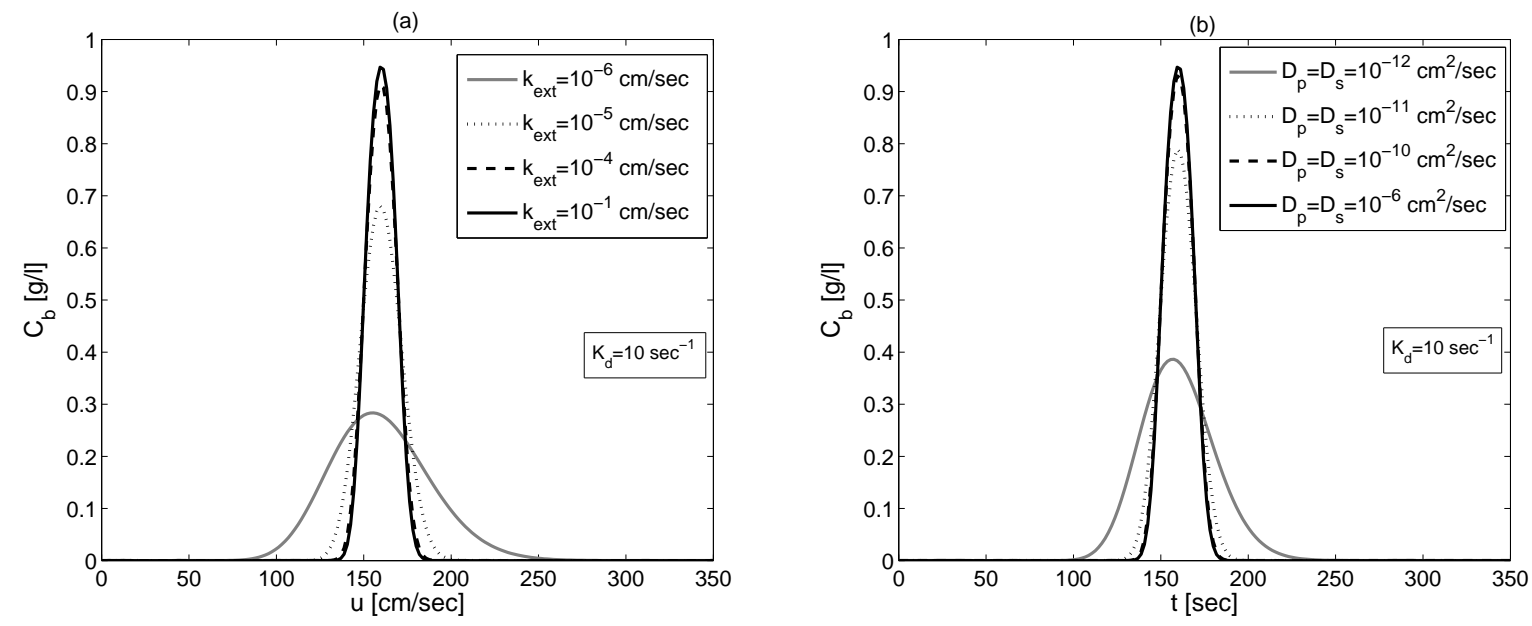

Figure 3: Effect of $k_{\text {ext }}$ and $D_{p}=D_{s}$ on the concentration profiles for fixed $K_{d}=10 \mathrm{sec}^{-1}$. Other parameters are given in Table 1. 

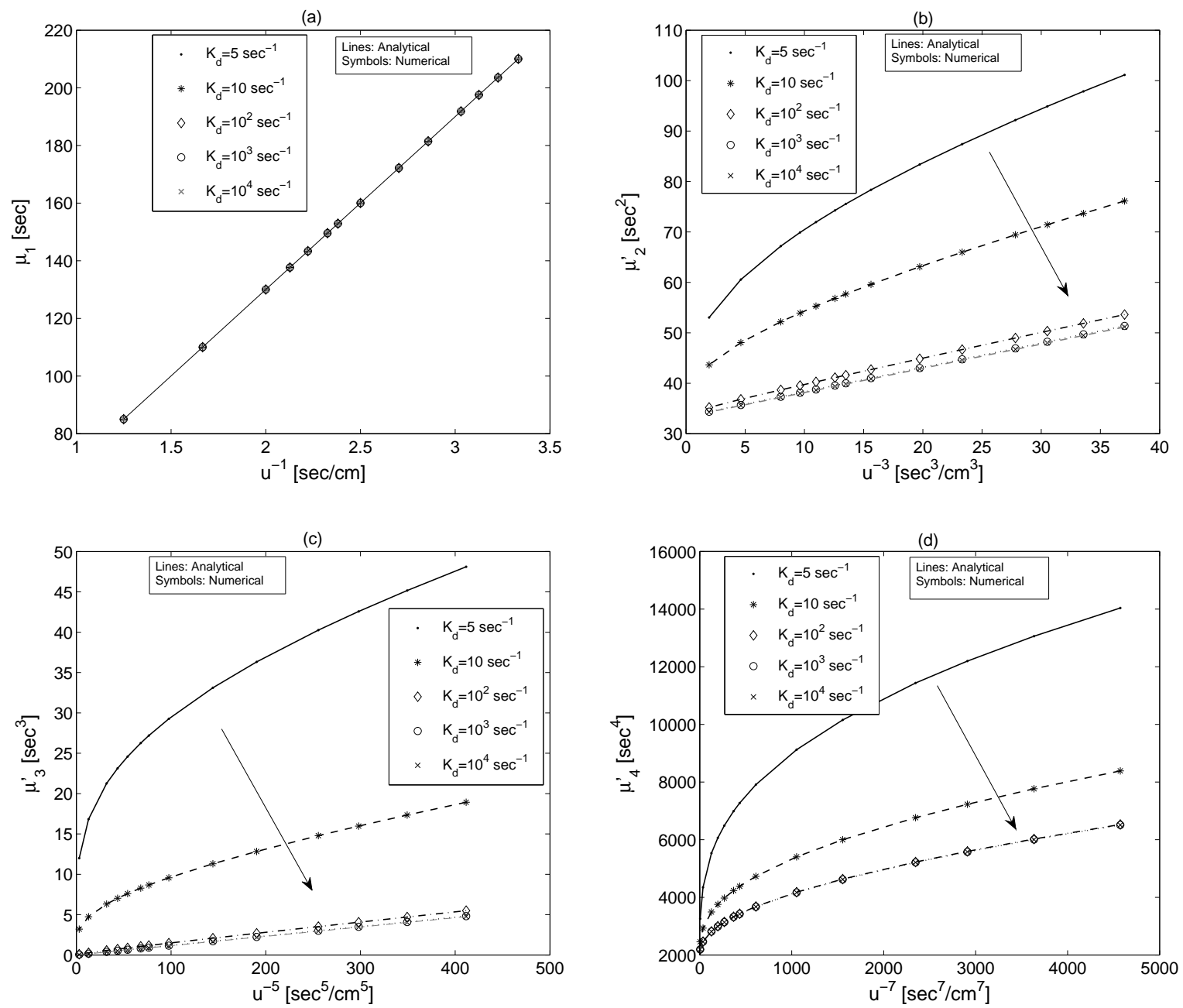

Figure 4: Effect of $K_{d}$ on temporal moments. Other parameters are given in Table 1. 
(a)

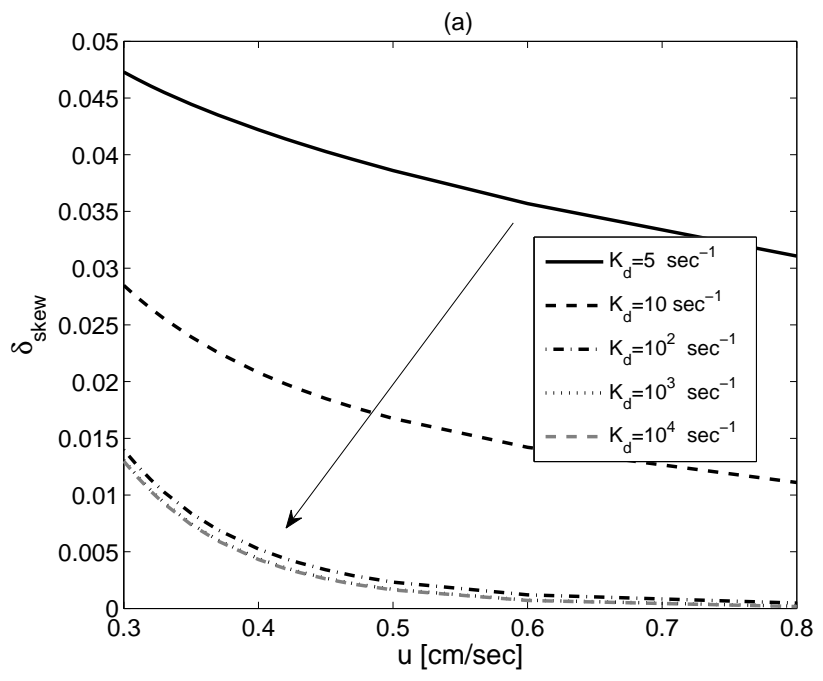

(b)

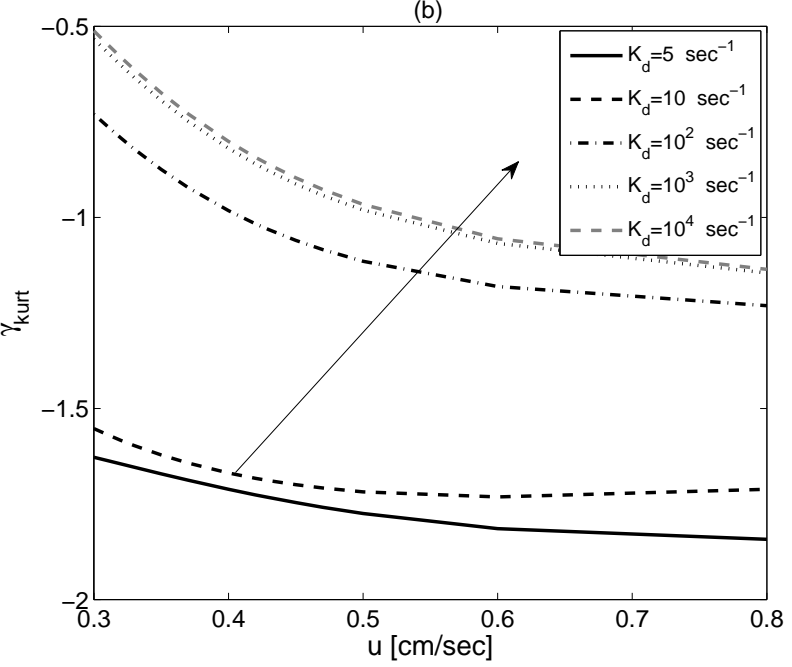

Figure 5: Effect of $K_{d}$ on the skewness and kurtosis. Other parameters are given in Table 1.
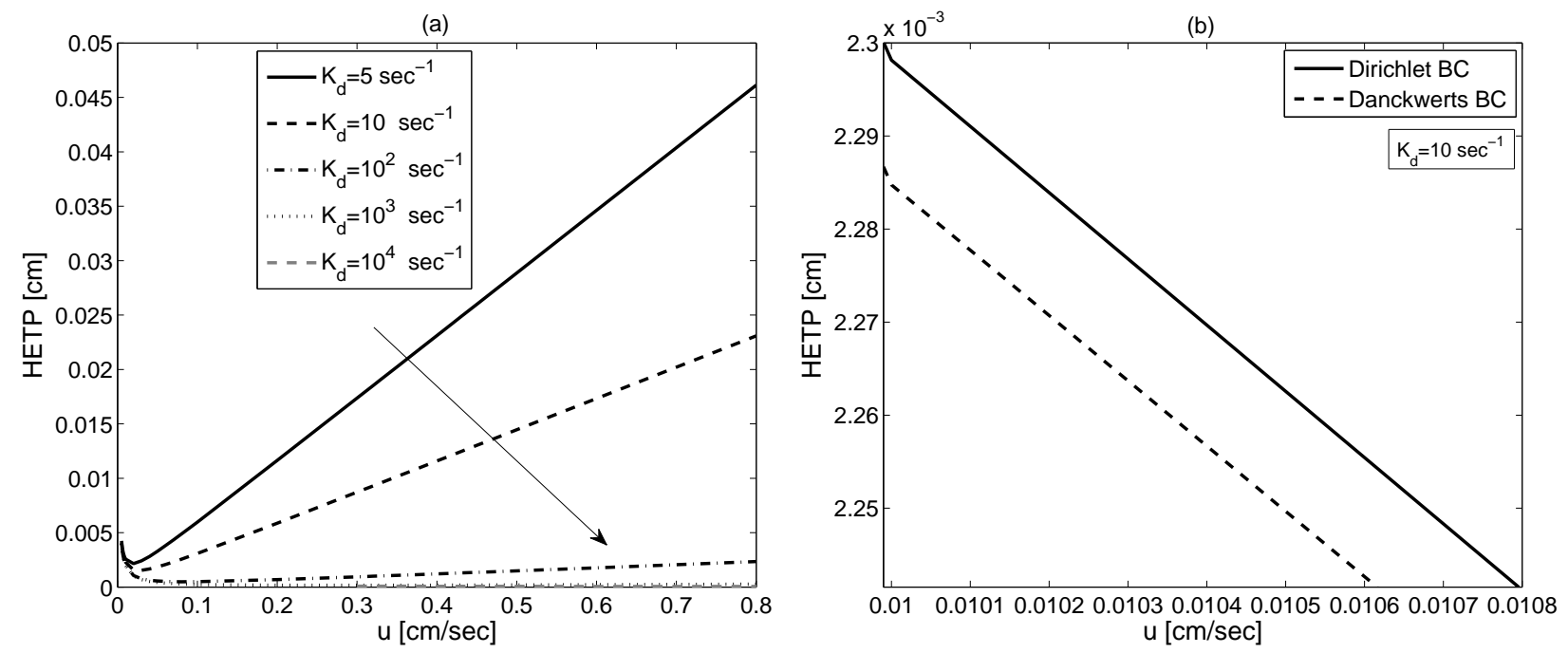

Figure 6: (a) Effect of $K_{d}$ on the HETP curve, (b) effect of BCs on the HETP curve. Other parameters are given in Table 1. 